\title{
Host selection by the louse fly Crataerina pallida; an avian nest ectoparasite of the common swift Apus apus
}

MARK D. WALKER and IAN D. ROTHERHAM

Parasitology (2010), doi:10.1017/S0031182010000740. Published by Cambridge University Press, 14 June 2010.

It has been brought to our attention that there may be rights issues in relation to the above article, which was published in FirstView on 14 June 2010. As a consequence, the article has been removed from FirstView and it will not be published in the print edition of Parasitology or on Cambridge Journals Online. 